\title{
Electroencephalography During the Acute Phase Of Encephalitis: A Brief Review
}

\author{
Leonardo Jardim Vaz de Mello ${ }^{\mathrm{a}, \mathrm{d}}$, Ali Seifi ${ }^{\mathrm{b}}$, Ilse Amairani Perez ${ }^{\mathrm{c}}$, \\ Daniel Agustin Godoy b, c
}

\begin{abstract}
Electroencephalography (EEG) remains an important complementary tool to diagnose neurologic diseases, including encephalitis. The objectives of this short review are to show the electroencephalographic findings in different types of encephalitis and to highlight the changing outlines during and after the specific treatment. In our study, we did a non-systematic review of literature on the role of EEG in infectious and autoimmune encephalitis. We concluded that EEG can be very useful in the diagnosis and follow-up of different types of encephalitis. Early changes during the illness can have an impact on prognosis.
\end{abstract}

Keywords: Electroencephalography; Encephalitis; Infectious; Autoimmune

\section{Introduction}

Electroencephalography (EEG) is an important diagnostic tool to evaluate and treat different types of epilepsy and other neurological conditions [1]. The surface EEG shows electrophysiological recordings of the cerebral cortex activity at any given time. Continuous information can be obtained with modern EEG monitoring techniques especially in acute and critical conditions [1]. In the context of encephalitis, in addition to detecting seizure activity, EEG helps to establish diagnosis [2, $3]$. In most cases, findings are nonspecific and denote global compromise of the brain funtion, but certain patterns are associated with certain etiologies. In addition, EEG provides information about prognosis and therapeutic response $[2,3]$. In

\footnotetext{
Manuscript submitted January 23, 2020, accepted February 24, 2020

a Jefe del Departamento de Neurologia, Universidad Federal de Sao Joao del Rei, Minas Gerais, Brazil

bUniversity of Texas Health Science Center at San Antonio, TX, USA

'University Hospital System of San Antonio, TX, USA

${ }^{\mathrm{d} C}$ Corresponding Author: Leonardo Jardim Vaz de Mello, Departmento de Neurologia, Universidad Federal de Sao Joao del Rei, Plaza Frei Orlando 170, Centro Sao Joao del Rei, Minas Gerais, CEP: 36307-352, Brazil.

Email: ljvmello@ufsj.edu.br
}

doi: https://doi.org/10.14740/jnr568 this brief review, we will review the usefulness and correlation between the main causes of encephalitis and their respective electroencephalographic findings.

\section{Definitions}

According to the guidelines of the International Encephalitis Consortium, the diagnosis requires the presence of encephalopathy (major criterion), two minor criteria for possible encephalitis and three or more for confirmation of encephalitis [4].

Encephalopathy is characterized by alterations in the level of consciousness, or changes in personality or behavior for more than $24 \mathrm{~h}$ without an obvious justifiable cause.

Minor criteria include the following: 1) fever $\geq 38{ }^{\circ} \mathrm{C}$ $\left(100.4^{\circ} \mathrm{F}\right)$ within the $72 \mathrm{~h}$ before presentation; 2$)$ generalized or partial seizures not attributable to a pre-existing seizure disorder; 3) focal neurological deficit; 4) white blood cell count $\geq 5 / \mathrm{mm}^{3}$ in cerebrospinal fluid (CSF); 5) abnormality of brain $\mathrm{pa}^{\mathrm{r}}$ enchyma on neuroimaging suggestive of encephalitis; and 6) changes in EEG that are consistent with encephalitis and not attributable to another cause.

\section{Etiology}

It is often difficult to establish the exact cause of encephalitis, but the most common cause is a viral infection. Encephalitis can be divided into primary and secondary. Primary encephalitis occurs when a virus or another agent directly colonizes the brain parenchyma. Infection can be localized in a specific geographic area or region or be widespread and globally spread throughout. Among the known and most common viruses that cause encephalitis, we can mention: herpes simplex virus (HSV) type 1 and 2, Epstein-Barr virus and enterovirus. Retrovirus are a large and important family of causative agents, including West Nile virus (WNV), chikungunya, dengue and tick-borne encephalitis viruses [2-5]. Secondary encephalitis results from an abnormal reaction of the immune system to an infection in another part of the body. These are called noninfectious or autoimmune encephalitis. They usually follow a subacute course, and generally symptoms start a few weeks after the initial infection and have detected several specific markers or antibodies. 
The most frequent autoimmune encephalitis is the anti-Nmethyl-D-aspartate (NMDA) receptor encephalitis [2-5], generally associated with an unknown neoplasm (ovary). It is also described within other entities such as paraneoplastic encephalitis. In around $60-75 \%$ of patients, neurological symptoms precede the diagnosis of malignant neoplasm [2-5].

\section{Risk Factors}

Extreme old age, defects or alterations of the immune system, neoplasms, specific geographical regions and certain seasons of the year are the main risk factors described for the development of encephalitis [2-5].

\section{Clinical Picture and Images}

Fever, alteration in the level of consciousness and seizures make up the classic triad of acute viral encephalitis; however, it is not always presented as such [2-5]. Acute confusional stateor atypical personality disorders are common. In autoimmune and limbic encephalitis, there are variations with respect to the diagnostic criteria [2-7]. In the case of autoimmune origin, at least three of the following criteria must be met: 1) subacute presentation of memory compromise, impaired consciousness, or psychiatric symptoms; 2) seizures in the absence of epilepsy; 3) a CSF pleocytosis $>4$ cells $/ \mathrm{mm}^{3}$; 4) neuroimaging suggestive of encephalitis, preferably magnetic resonance imaging (MRI) (high signal intensity in T2 or fluid attenuated inversion recovery (FLAIR) MR images, located mainly in medial temporal lobes together with multifocal compromise of gray and/or white matter, compatible with demyelination or inflammatory process) $[6,7]$.

\section{Detection of Specific Markers in Non-Infectious Encephalitis}

Different types of antibodies responsible for autoimmune encephalitis have been detected. These can be directed against intracellular antigens, especially when they are associated with neoplastic disease, or they can attack surface proteins, in which case the prognosis is more favorable [5-8]. Among the neoplasms involved in the development of autoimmune encephalitis, we can highlight: small cell lung carcinoma (anti-Hu antibodies, anti- $\alpha$-amino-3-hydroxy-5-methyl4-isoxazolepropionic acid, anti-AMPAR, anti-collapsin, CRMP5), anti-amphiphysin; testicular germ cell tumors (anti$\mathrm{Ma}$ /Ta), thymoma (anti-CRMP5), breast cancer (anti-amphiphysin) and Hodgkin lymphoma [5-8]. Often the tumor has not been previously diagnosed [5-8].

\section{EEG in Encephalitis}

Finding a specific electroencephalographic pattern for encephalitis remains a challenge, taking into account the various existing etiologies [1]. In HSV encephalitis, perhaps the most analyzed encephalitis in the literature, EEG can show unilateral or bilateral periodic discharges together with the presence of focal or generalized slow waves, and electrical discharges, which shows that usually there is no specific pattern that identifies it as such [1-3]. During herpetic encephalitis, periodic high-voltage acute waves can be observed, together with the presence of complex and sharp wave potentials or also at the point of periodic appearance [1-4]. In general, the changes are not global but are located on the side where the engagement occurs, usually the temporal lobes.

Figure 1 shows EEG characteristic but nonspecific changes in herpetic encephalitis. The first arrow (a) shows periodic wave point complexes from the right side to temporal predominance (circle). The second arrow (b) shows a slow base rhythm (theta-delta) together with synchronous bilateral potentials formed by acute waves with a predominance on the right side (arrow).

The presence of three-phase waves is common but nonspecific. They occur in multiple encephalopathies and metabolic alterations of various conditions, such as hepatic, uremic and septic encephalopathies, but may also be present during the course of viral encephalitis or another etiology $[1,9]$.

Figure 2 shows the presence of bilateral slow thriphasic waves in a patient affected by West Nile encephalitis. This trace is similar to referenced in literature [10].

Another situation where we can find the presence of threephase waves is during the invasion of the brain parenchyma by prions as in the case of Creutzfeldt-Jakob disease [11].

In autoimmune encephalitis, the most studied type is encephalitis due to anti-NMDA antibodies. The presence of extreme delta brush pattern in the EEG strongly suggests a diagnosis $[12,13]$. The EEG record in Figure 3 demonstrates a generalized activity of rhythmic and semi-rhythmic delta frequency at $1 \mathrm{~Hz}$ with superimposed bursts, predominantly frontal of rhythmic beta frequency activity $[12,13]$. This finding reinforces the diagnosis of NMDAR encephalitis, but its real value is still unknown.

Multiple autoimmune encephalitis, either seropositive or negative, show different types of unspecific EEG alterations such as: frontal intermittent rhythmic delta activity (FIRDA), periodic lateralized epileptiform discharge (PLED), periodic epileptiform discharges (PED), generalized rhythmic delta activity (GRDA), excess beta activity (EBA) and triphasic waves [14-18]. An example is showed in Figure 4.

\section{Continuous EEG Monitoring}

This is a modality of non-invasive monitoring of brain function, especially in critically ill patients or under deep sedoanalgesia [1]. Its utility is increased day by day and is not limited to the detection of seizure activity. Continuous EEG monitoring in its quantitative modality (qEEG) provides real-time information [1]. The qEEG is a digitalized alternative of EEG analysis that allows the graphic exposure of changes in brain frequencies over time. This system is based on mathematical algorithms, which transform the conventional EEG into a com- 


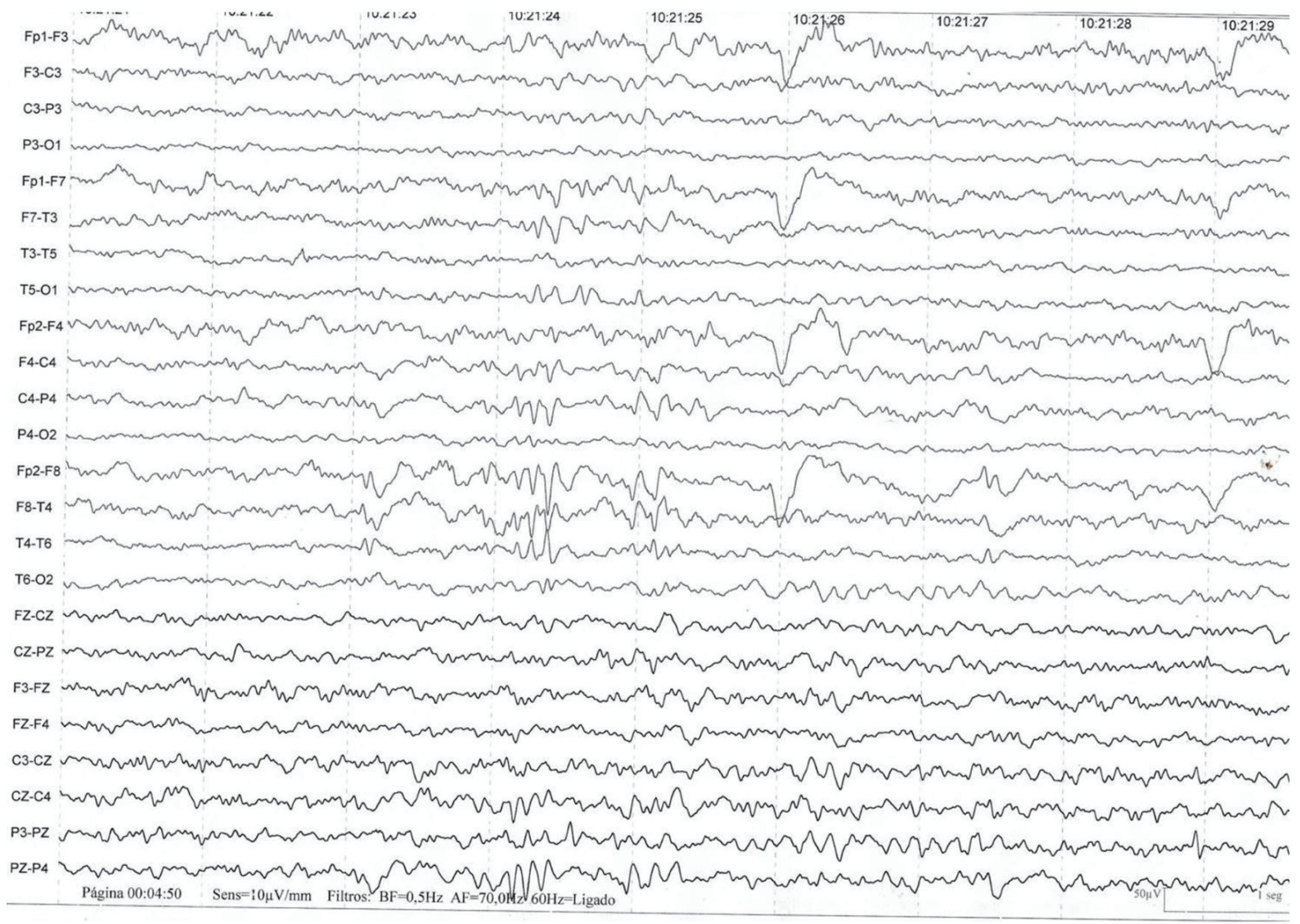

Figure 1. The presence of three-phase waves are common but nonspecific. They occur in multiple encephalopathies and metabolic alterations of various conditions, such as hepatic, uremic and septic encephalopathies, but may also be present during the course of viral encephalitis or another etiology $[1,9]$.

pact one, representing the different waves graphically using colors [1]. Thus, several hours of regular EEG can be reduced by just a simple screen view.

The utility of qEEG monitoring is not limited to the detection of seizure discharges but provides very useful information when assessing the evolution of the disease, its response to the instituted treatment including the graduation of the sedation level and the detection of undesirable effects of certain drugs including antibiotics. It also has prognostic value [1] (Fig. 5).

\section{Conclusion}

EEG is an important diagnostic and monitoring tool to consider in the management of different types of encephalitis. There is no pathognomonic EEG pattern for any type of encephalitis. In general, viral encephalitis has slow background rhythms along with the presence of focal epileptogenic discharges. Autoimmune encephalitis due to anti-NMDA receptor antibodies characteristically, although not constantly, has an extreme delta brush pattern (EDB) frequently located in the frontal region. The EEG provides information that helps in determining the prognosis. Additionally, the EEG allows monitoring and response to therapy.

\section{Acknowledgments}

None to declare.

\section{Financial Disclosure}

None to declare.

\section{Conflict of Interest}

None to declare.

\section{Author Contributions}

Leonardo Jardim Vaz de Mello: bibliographic search and wrote the review. Ali Seifi: english edition. Ilse Amairani Perez: 


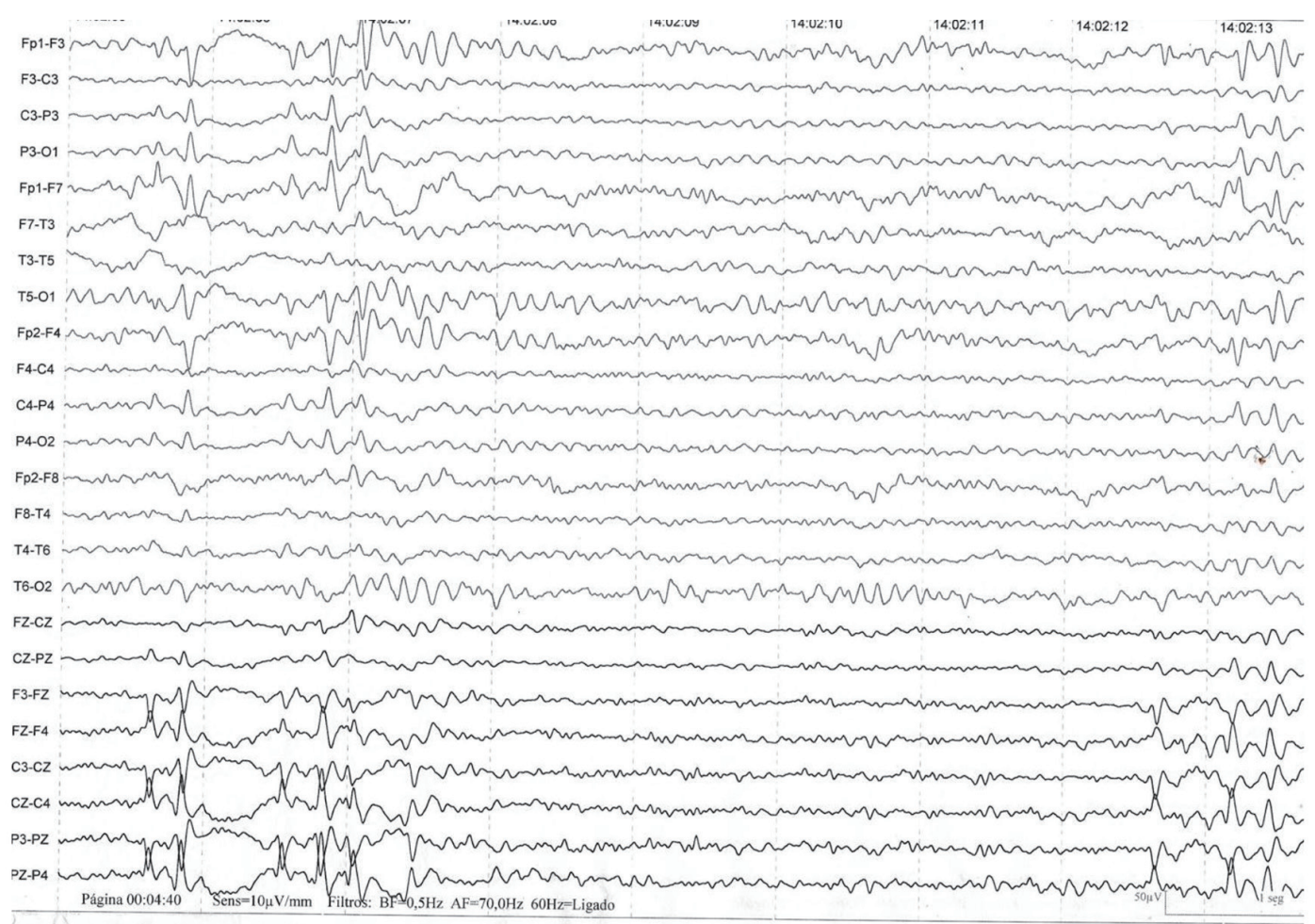

Figure 2. The presence of bilateral slow thriphasic waves in a patient affected by West Nile encephalitis. This trace is similar to referenced in literature [10].

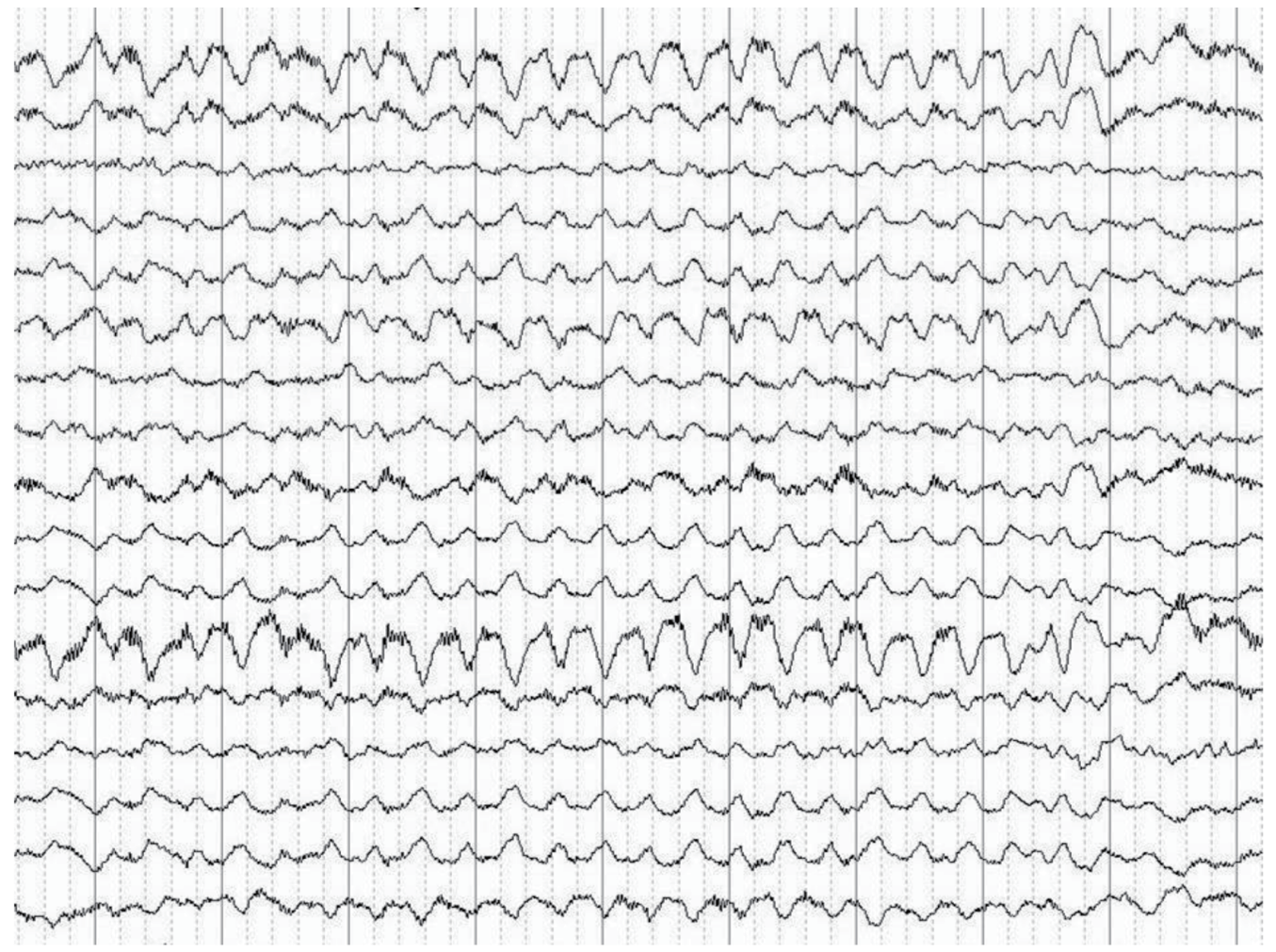

Figure 3. Generalized activity of rhythmic and semi-rhythmic delta frequency at $1 \mathrm{~Hz}$ with superimposed bursts, predominantly frontal of rhythmic beta frequency activity. 


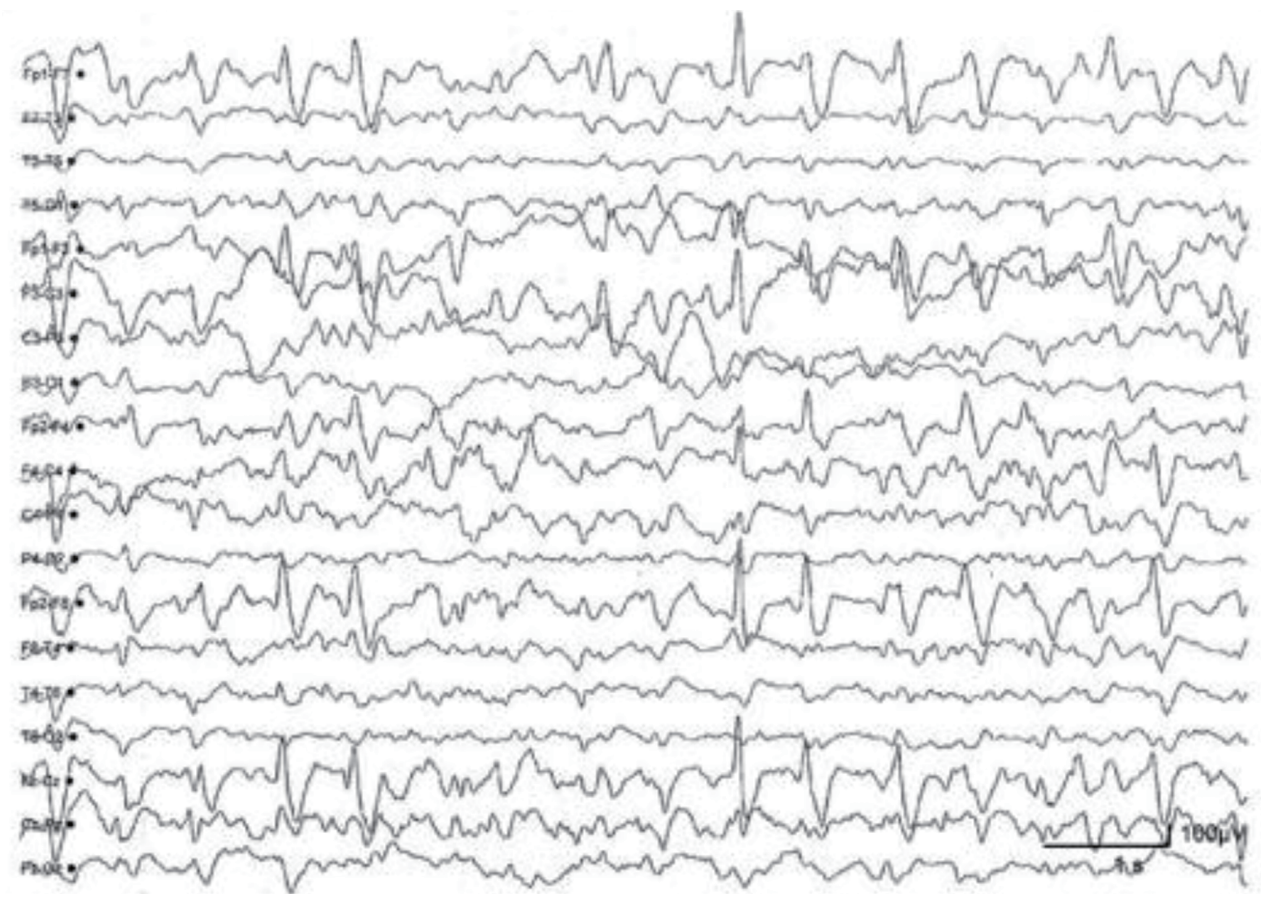

Figure 4. Anti-NMDA receptor encephalitis. Generalized irregular activity of 1.5 to $2 \mathrm{~Hz}$ peak and predominant wave in the previous regions of the hemisphere and slow and diffuse background activity. The record shows that the extreme delta brush pattern is not pathognomonic or unique sign of EEG in anti-NMDA receptor encephalitis. NMDA: N-methyl-D-aspartate; EEG: electroencephalography.

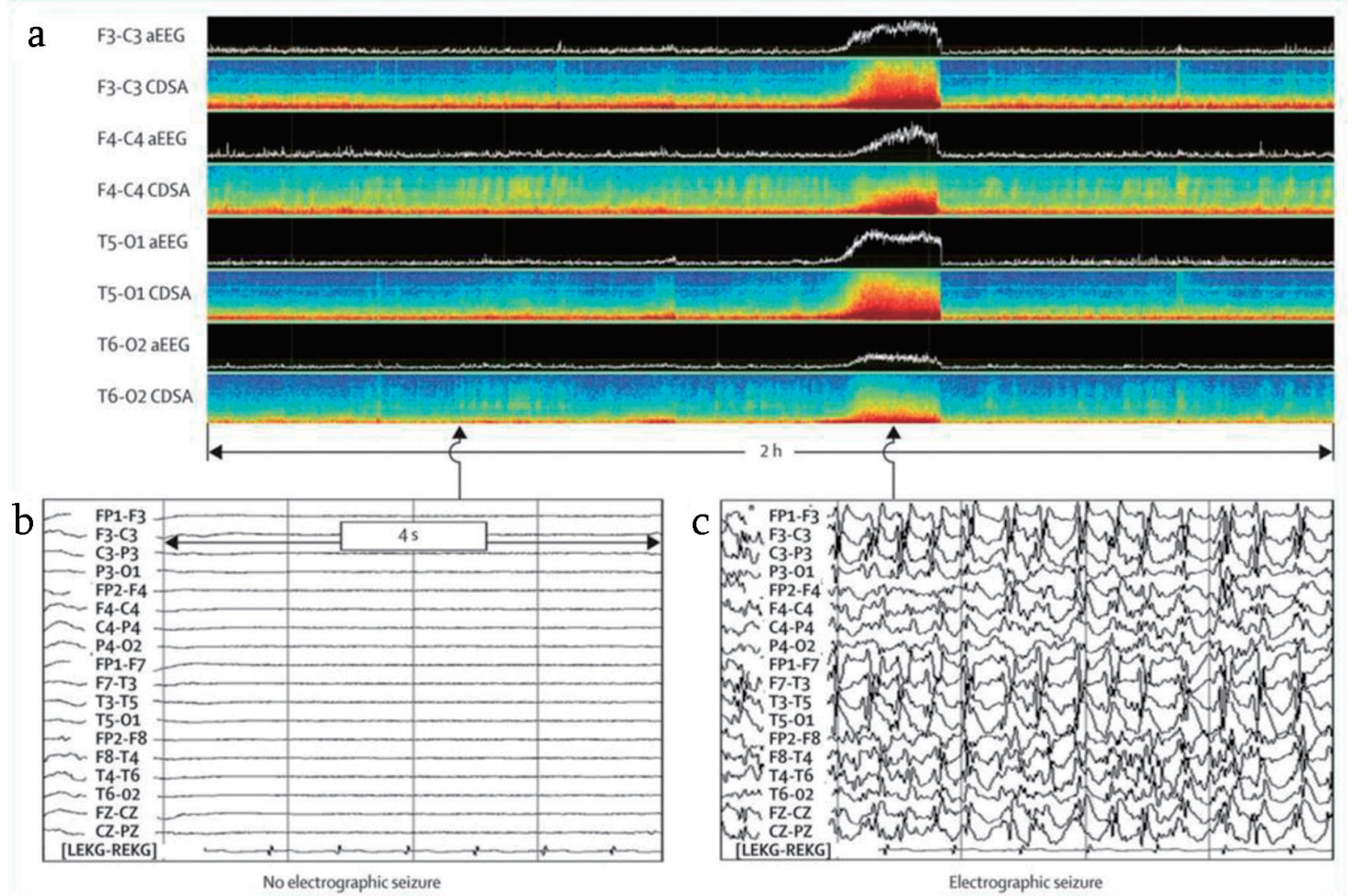

Figure 5. (a) qEEG monitoring showing the electrical activity in status epilepticus due to traumatic brain injury. (b) EEG showing no electroencephalographic seizure. (c) EEG showing diffuse electroencephalographic seizures. qEEG: quantitative electroencephalography. 
translate paper from Spanish to english. Daniel Agustin Godoy: intelectual author; corrections, edition of the paper ansd translation from portuguese to spanish

\section{Data Availability}

The authors declare that data supporting the findings of this study are available within the article.

\section{References}

1. Rubinos C, Godoy DA. Electroencephalographic monitoring in the critically ill patient: What useful information can it contribute? Med Intensiva. 2019. Epub 24 June 2019

2. Sutter R, Kaplan PW, Cervenka MC, Thakur KT, Asemota AO, Venkatesan A, Geocadin RG. Electroencephalography for diagnosis and prognosis of acute encephalitis. Clin Neurophysiol. 2015;126(8):1524-1531.

3. Halperin JJ. Chapter 18 - Diagnosis and management of acute encephalitis. In: Wijdicks EFM, Kramer AH (ed.), Critical Care Neurology Part I, Hand book of Clinical Neurology (3rd series), Vol. 140. Elsevier, 2017:337-347.

4. Venkatesan A, Tunkel AR, Bloch KC, Lauring AS, Sejvar J, Bitnun A, Stahl JP, et al. Case definitions, diagnostic algorithms, and priorities in encephalitis: consensus statement of the international encephalitis consortium. Clin Infect Dis. 2013;57(8):1114-1128.

5. Granerod J, Ambrose HE, Davies NW, Clewley JP, Walsh AL, Morgan D, Cunningham R, et al. Causes of encephalitis and differences in their clinical presentations in England: a multicentre, population-based prospective study. Lancet Infect Dis. 2010;10(12):835-844.

6. Pruss H, Dalmau J, Harms L, Holtje M, Ahnert-Hilger G, Borowski K, Stoecker W, et al. Retrospective analysis of NMDA receptor antibodies in encephalitis of unknown origin. Neurology. 2010;75(19):1735-1739.

7. Billinton A, Ige AO, Wise A, White JH, Disney GH, Marshall $\mathrm{FH}$, Waldvogel HJ, et al. GABA(B) receptor heterodimer-component localisation in human brain. Brain
Res Mol Brain Res. 2000;77(1):111-124.

8. Collao Parra JP, Urra CR, Derio CD. Encefalitis autoinmunes: criterios diagnosticos y pautas terapeuticas. Rev Med Chile. 2018;146:351-336.

9. Brodtkorb E, Lindqvist M, Jonsson M, Gustafsson A. Diagnosis of herpes simplex encephalitis. A comparison between electroencephalography and computed tomography findings. Acta Neurol Scand. 1982;66(4):462471.

10. Rodriguez AJ, Westmoreland BF. Eletroencephalografic Charactheristics of patient infected with West Nile Virus. J Clin Neurophysiol. 2007;24(5):386-389.

11. Shin JW, Yim B, Oh SH, Kim NK, Lee SK, Kim OJ. Redefining periodic patterns on electroencephalograms of patients with sporadic Creutzfeldt-Jakob disease. Clin Neurophysiol. 2017;128(5):756-762.

12. Mahawish K, Teinnert L, Cavanagh K, Brennan J. Limbic Encephalitis. BMJ Case Rep. 2014;2014:bcr2014204591

13. Schmitt SE, Pargeon K, Frechette ES, Hirsch LJ, Dalmau J, Friedman D. Extreme delta brush: a unique EEG pattern in adults with anti-NMDA receptor encephalitis. Neurology. 2012;79(11):1094-1100.

14. Foff EP, Taplinger D, Suski J, Lopes MB, Quigg M. EEG findings may serve as a potential biomarker for anti-NMDA receptor encephalitis. Clin EEG Neurosci. 2017;48(1):48-53.

15. Ueda J, Kawamoto M, Hikiami R, Ishii J, Yoshimura H, Matsumoto R, Kohara N. Serial EEG findings in antiNMDA receptor encephalitis: correlation between clinical course and EEG. Epileptic Disord. 2017;19(4):465470.

16. Baysal-Kirac L, Tuzun E, Altindag E, Ekizoglu E, Kinay D, Bilgic B, Tekturk P, et al. Are there any specific EEG findings in autoimmune epilepsies? Clin EEG Neurosci. 2016;47(3):224-234.

17. Qiu X, Zhang H, Li D, Wang J, Jiang Z, Zhou Y, Xu P, et al. Analysis of clinical characteristics and poor prognostic predictors in patients with an initial diagnosis of autoimmune encephalitis. Front Immunol. 2019;10:1286.

18. Shi Y. Serial EEG monitoring in a patient with antiNMDA receptor encephalitis. Clin EEG Neurosci. 2017;48(4):301-303. 